\title{
MELHORA DO ESTILO DE VIDA REDUZ O ÍNDICE DE CASTELLI 1 EM INDIVÍDUOS COM SÍNDROME METABÓLICA
}

\section{Ana Maria Pandolfo Feoli}

Doutora em Ciências Biológicas (UFRGS). Pontifícia Universidade Católica do Rio Grande do Sul (PUCRS), Brasil.

\section{Édina Caroline Ternus Ribeiro}

Nutricionista. Pontifícia Universidade Católica do Rio Grande do Sul (PUCRS), Brasil.

\section{Carla Haas Piovesan}

Doutora em Medicina e Ciências da Saúde PUCRS Pontifícia Universidade Católica do Rio Grande do Sul (PUCRS), Brasil.

\section{Fabrício Edler Macagnan}

Doutor em Ciências da Saúde pela Pontifícia Universidade Católica do Rio Grande do Sul. Universidade Federal de Ciências da Saúde de Porto Alegre (UFCSPA), Brasil.

\section{Margareth Oliveira}

Doutora em Psiquiatria e Psicologia Médica pela Universidade Federal de São Paulo. Pontifícia Universidade Católica do Rio Grande do Sul (PUCRS), Brasil.

\section{Andréia da Silva Gustavo}

Doutora em Epidemiologia UFRGS. Pontifícia Universidade Católica do Rio Grande do Sul (PUCRS), Brasil.
RESUMO: Avaliar o efeito de diferentes intervenções para modificação do estilo de vida sobre o Índice de Castelli 1 em indivíduos com síndrome metabólica. Tratou-se de uma subanálise de um ensaio clínico randomizado. Foram incluídos no estudo indivíduos com diagnóstico de síndrome metabólica e idade entre 30 e 59 anos, que foram posteriormente randomizados em três diferentes grupos de intervenção. Foram realizadas avaliações antropométricas e bioquímicas no início do estudo e ao final dos três meses de intervenção. Participaram do estudo 59 indivíduos. Os modelos de intervenção realizados nos grupos intervenção individual e intervenção em grupo foram eficazes na redução significativa do índice de Castelli 1 , com variância absoluta de $-0,70$ e $-0,23$, respectivamente. $O$ modelo de intervenção padrão não demonstrou redução favorável. As intervenções que contemplaram acompanhamento multiprofissional produziram efeito benéfico na redução do índice de Castelli 1 em pacientes com síndrome metabólica.

PALAVRAS-CHAVE: Síndrome Metabólica; Estilo de vida; Fatores de risco; Doenças cardiovasculares.

\section{LIFEST YLE IMPROVEMENT REDUCES CASTELLI 1 IN- DEX IN PEOPLE WITH METABOLIC SYNDROME}

\begin{abstract}
The effect of different interventions for lifestyle modification on Castelli I Index in people with metabolic syndrome is evaluated. It is a sub-analysis of a randomized clinical assay. People with metabolic syndrome, aged between 30 and 59 years old, were included and later divided, at random, into three different intervention groups. Anthropometric and biochemical evaluations of 59 people were performed at the start and at the end of the three months of intervention. Intervention models undertaken in individual intervention groups and intervention in groups were efficient to significantly reduce Castelli 1 Index, with absolute variance of -0.70 and -0.23 , respectively. Standard intervention model did not reveal favorable reduction. Interventions that underscored multiprofessional following up produced beneficent effect on the reduction of Castelli 1 Index in patients with metabolic syndrome.
\end{abstract}

KEY WORDS: Metabolic Syndrome; Life style; Risk factors; Cardiovascular diseases. 


\section{INTRODUÇÃO}

A Síndrome Metabólica (SM) é uma condição clínica descrita como um conjunto de desordens metabólicas que elevam os fatores de risco cardiovascular relacionados à deposição central de gordura e à resistência à insulina. A presença desta síndrome pode aumentar relativamente a mortalidade geral, mas tem efeito predominantemente sobre a morte por causas cardiovasculares ${ }^{1-3}$.

Atualmente a SM se apresenta como um problema de saúde pública, com elevada prevalência mundial. Estima-se que $25 \%$ a $35 \%$ da população global possuam diagnóstico de SM, fato que está intimamente ligado ao aumento das prevalências dos componentes que definem a $\mathrm{SM}^{4}$, como a obesidade, que tem sido apontada como principal fator desencadeante de desordens metabólicas, e que também possui alta prevalência, já que no Brasil cerca de 30\% dos indivíduos é classificado como obeso5.

Modificar os hábitos de vida pode promover benefícios importantes para os portadores dessa síndrome. Espera-se que com a adesão a hábitos de vida considerados saudáveis preconizados como estratégias não farmacológicas recomendadas para o tratamento da síndrome metabólica ${ }^{1}$, como prática regular de exercício físico e alimentação balanceada, ocorra redução nos valores de colesterol total, triglicerídeos, glicemia de jejum, pressão arterial, peso e circunferência do abdome ${ }^{1}$. Detectar os processos que desencadeiam o excesso de peso, a dislipidemia, o aumento da pressão arterial e a glicemia de jejum estão dentre as metas priorizadas para a redução do risco cardiovascular (RCV) $)^{6.9}$.

Indivíduos com o diagnóstico de SM necessitam de rastreamento de RCV e acompanhamento da evolução dos critérios de diagnóstico para monitorar o efeito das intervenções realizadas. A utilização de escores que permitem a estratificação de risco é importante tanto na prevenção quanto no tratamento dessa condição clínica, tendo em vista a multiplicidade de fatores de risco cardiovasculares que possam estar presentes nesses indivíduos ${ }^{3}$ 10 .

O índice de Castelli 1 (IC-1) é um escore que permite identificar indivíduos com risco aumentado para eventos cardiovasculares importantes, como o infarto agudo do miocárdio (IAM). O IC-1 é calculado a partir da razão entre o colesterol total e a fração de colesterol de alta densidade (HDL-c). O resultado deste escore pode ser utilizado também para demonstrar o efeito de uma intervenção voltada para mudanças de estilo de vida ${ }^{11,12}$.

Sendo assim, diante do exposto, o objetivo deste trabalho foi analisar os efeitos de diferentes modelos de intervenção para modificação do estilo de vida sobre o IC-1 de indivíduos com SM.

\section{METODOLOGIA}

Este estudo é uma subanálise de um ensaio clínico randomizado, que foi realizado com os dados coletados pelo estudo principal, denominado: efeito de diferentes intervenções de modificação do estilo de vida sobre aspectos físicos, metabólicos e comportamentais envolvidos na síndrome metabólica.

Os participantes foram selecionados a partir da divulgação em mídia impressa. Foram incluídos no estudo indivíduos de ambos os sexos entre 30 e 59 anos, diagnosticados para SM, conforme o National Cholesterol Education Program Adult Treatment Panel III (NCEP-ATP III) ${ }^{13}$, que consiste na presença de três ou mais dos seguintes critérios: circunferência abdominal $\geq 88 \mathrm{~cm}$ para mulheres e $\geq 102 \mathrm{~cm}$ para homens; pressão arterial sistólica (PAS) $\geq 130 \mathrm{mmHg}$ e diastólica $(\mathrm{PAD}) \geq$ $85 \mathrm{mmHg} ; \mathrm{GJ} \geq 100 \mathrm{mg} / \mathrm{dL}$; triglicerídeos $\geq 150 \mathrm{mg} / \mathrm{dL}$; e HDL-c $<40 \mathrm{mg} / \mathrm{dL}$ para homens e $<50 \mathrm{mg} / \mathrm{dL}$ para mulheres. Não fizeram parte do estudo os indivíduos que apresentaram uma ou mais das seguintes situações: contraindicação absoluta para exercício físico por problemas musculoesqueléticos, neurológicos, vasculares, pulmonares e cardíacos; uso de hipolipemiantes; uso de anticoagulante; praticantes de exercício físico regular (a partir de 30 minutos 02 vezes na semana); portadores de distúrbios psiquiátricos ou usuários de medicação antidepressiva; diagnóstico de hipotireoidismo; gestantes; usuários de qualquer outro suplemento alimentar e vitamínico; dificuldade de contato e/ou incapacidade de retorno e acompanhamento. Os indivíduos foram randomizados pelo estudo principal, em três modalidades de intervenção do estilo de vida: (1) Intervenção Padrão (IP): a intervenção não medicamentosa recomendada 
nas principais diretrizes para o manejo clínico da SM foi denominada de intervenção padrão de modificação do estilo de vida que, nesse estudo, foi considera como grupo controle. Os voluntários desse grupo participaram de duas consultas, uma no momento de entrada na pesquisa e outra após três meses. As consultas foram realizadas pela equipe de enfermagem. Na primeira consulta os participantes foram orientados quanto à prática de exercício físico, ao autocuidado e à alimentação $0^{14}$. Em relação ao autocuidado, houve orientação e reforço quanto aos cuidados para a administração dos medicamentos em uso e cuidados com a saúde em geral. Na última consulta foram abordadas as facilidades e dificuldades, em seguir as orientações de modificação dos hábitos alimentares e a prática regular de exercício físico; (2) Intervenção em Grupo (IG): Nessa modalidade de intervenção, a motivação para a mudança do estilo de vida foi trabalhada em grupo. Os encontros do grupo foram realizados na presença de um membro da equipe da psicologia, enfermagem, fisioterapia e nutrição. A duração dos encontros foi de 1 hora e 45 minutos. Nos primeiros 45 minutos, foi abordado um tema em saúde com o objetivo trabalhar temas predefinidos de educação em saúde. Todos os temas foram focados nos principais fatores de risco cardiovasculares considerados modificáveis e que estão associados à $\mathrm{SM}^{1}$. Nos 60 minutos finais, a motivação para mudança de comportamento foi trabalhada com base no modelo transteórico de mudança ${ }^{15}$ o qual propõe uma estrutura para compreender e intervir junto ao paciente de acordo com o estágio motivacional em que se encontra. Neste segundo momento do grupo, foram discutidas e testadas estratégias de modificação dos hábitos relacionados à alimentação e a prática regular de atividade física possíveis de serem incorporadas nas rotinas diárias dos voluntários. (3) Intervenção Individual (II): Essa modalidade de intervenção baseia-se em uma proposta de atendimento ambulatorial de modificação do estilo de vida de indivíduos com SM. Os voluntários desse grupo participaram de consultas semanais individualizadas com a equipe de nutrição, psicologia e realizaram prática regular de exercício físico com a equipe de fisioterapia. A intervenção nutricional proposta para este grupo foi individualizada $e$ baseada nas necessidades de cada participante. Os voluntários receberam um plano alimentar e foram orientados a desenvolver um plano de ação alimentar voltados para os aspectos que devem ser modificados. As estratégias foram formuladas individualmente respeitando-se as condições intrínsecas e extrínsecas necessárias para o processo de modificação dos hábitos alimentares. Durante as consultas semanais foi realizado acompanhamento do peso corporal e da adesão ao plano alimentar por meio de um breve inquérito recordatório de 24 horas ${ }^{16,17}$. Além disso, foram constantemente monitoradas e registradas as possíveis dificuldades em aderir às estratégias e metas combinadas na consulta anterior. Junto a essas ações, temas relacionados à SM foram desenvolvidos a partir em um roteiro pré-definido. As consultas semanais com a psicologia foram baseadas no modelo transteórico de mudança, adaptado para a modalidade individual ${ }^{15}$, o qual trabalha os diferentes estágios de mudança assim como os processos de mudança específicos. As sessões seguiram um roteiro estruturado com objetivos pré-definidos com base nos processos de mudança e estágios de motivação específicos. O programa de exercício físico, acompanhado pela equipe da fisioterapia, consistia de 36 sessões em esteira rolante. Cada sessão teve a duração de 60 minutos contínuos, três vezes por semana, com intensidade ajustada pela frequência cardíaca (FC). A faixa de treinamento ficou entre $75 \%$ a $85 \%$ da FC de máxima avaliada no teste incremental de membros inferiores (TIMI) ${ }^{14}$. Durante o exercício físico foram monitoradas a $\mathrm{PA}, \mathrm{FC}$ e os sinais e sintomas de alterações cardiovasculares. A velocidade e inclinação da esteira foram constantemente ajustadas a fim de manter a FC dentro da faixa de treinamento.

Todos os grupos foram avaliados no início e ao final das intervenções (após 03 meses). A avaliação antropométrica contemplou a aferição do peso corporal, da altura e da circunferência abdominal. O peso corporal foi verificado por meio de uma balança da marca Filizola ${ }^{\circledR}$, com capacidade para $160 \mathrm{~kg}$, devidamente calibrada, com o paciente descalço e com o mínimo de roupa possível. Para a mensuração da estatura, utilizou-se um antropômetro vertical (marca Sunny®). A circunferência abdominal foi avaliada por meio do perímetro abdominal (cm), aferido no local de máxima extensão da região do abdome ${ }^{18}$. O instrumento utilizado foi uma fita milimetrada inelástica com $180 \mathrm{~cm}$ de comprimento. $\mathrm{O}$ cálculo e a classificação do IMC foram realizados de acordo com a 
Organização Mundial da Saúde (OMS) ${ }^{19}$.

Os valores da PAS e da PAD foram aferidos de acordo com as recomendaçóes preconizadas pela $7^{\mathrm{a}} \mathrm{Di}$ retriz Brasileira de Hipertensão Arteria ${ }^{20}$. Os marcadores bioquímicos analisados foram o perfil lipídico (colesterol total, HDL-c e triglicerídeos) e a glicemia de jejum. Para a análise desses marcadores, foram coletados $10 \mathrm{~mL}$ de sangue de cada participante, através de punção venosa, após 12 horas de jejum, antes e após os três meses de intervenção. A determinação do LDL-colesterol (LDL-c) foi realizada de forma indireta, utilizando-se equação de Friedewald ${ }^{21}$.

Para a avaliação do IC-1, foi utilizada a fórmula original proposta por Castelli ${ }^{12}$, na qual se utiliza a razão entre o colesterol total e o HDL-c (IC1 = colesterol total/ HDL-c), a partir dos valores plasmáticos de colesterol total e HDL-c, ambos em mg/dL; esse cálculo foi realizado antes da intervenção e após três meses de acompanhamento. O Quadro 1, a seguir, apresenta os valores de referência desse critério.

Quadro 1. Valores de referência para o Índice de Castelli 1. DCV: Doença Cardiovascular

\begin{tabular}{|c|c|c|}
\hline \multirow{2}{*}{ HOMENS } & Valor de referência & Classificação \\
\cline { 2 - 3 } & $<5,1 \mathrm{mg} / \mathrm{dL}$ & Baixo risco DCV \\
\hline \multirow{2}{*}{ MULHERES } & $<5,1 \mathrm{mg} / \mathrm{dL}$ & Alto risco DCV \\
\cline { 2 - 3 } & $>4,4 \mathrm{mg} / \mathrm{dL}$ & Baixo risco DCV \\
\hline
\end{tabular}

Para a análise estatística foi utilizado o programa Statistical Package for the Social Sciences - SPSS, versão 17.0. Inicialmente, realizou-se o teste $T$ de Student para amostras em pares, que consistiu em avaliar os valores do IC-1 antes e após o acompanhamento intragrupo, buscando verificar a variação $(\Delta)$ sem fazer correlações com as demais intervenções. Em um segundo momento, foi utilizado o Post Hoc Test de Tukey HSD, a partir do One-Way ANOVA, em que se comparou a variação da diferença entre escores de cada grupo antes e após a intervenção $(\Delta)$, para realizar os testes de comparações múltiplas entre eles, verificando o efeito de cada grupo. Adotou-se o nível de significância ( $\alpha$ ) de 5\% como critério de decisão. As informações referentes aos procedimentos envolvidos no estudo foram fornecidas individualmente, por meio do termo de consentimento livre e esclarecido (TCLE), específico para cada intervenção. O estudo foi aprovado pelo Comitê de Ética da PUCRS sob o processo de $n^{\circ} 10 / 05153$ e cadastrado no Registro Brasileiro de Ensaios Clínicos, ReBEC, sob número: RBR-9wz5fc.

\section{RESULTADOS}

A amostra avaliada foi constituída por 59 indivíduos, com média de idade de 51,4 anos, sendo a maioria mulheres, perfazendo $54,2 \%$ da amostra. A caracterização da amostra está descrita na tabela 1. Os grupos foram considerados homogêneos para todas as variáveis consideradas.

Na Tabela 2, estão os resultados do IC-1 mensuradas nos momentos pré e pós-intervenção. As variações absolutas e percentuais também estão descritos e mostram que apenas os grupos IG e II demonstraram melhora significativa, enquanto que no grupo IP os resultados não foram favoráveis.

No teste de comparação de amostras entre pares, os grupos II e IG mostraram efeito semelhante na redução do IC-1. Já o IP, não mostrou efeito na redução do índice, quando comparado com II e IG.

Na Tabela 3, estão descritos os resultados da classificação do IC-1 por gênero antes e após as diferentes intervenções. Houve mudança na média dos escores de classificação atribuídos às mulheres do grupo II e aos homens do grupo IG, que passaram de alto risco para baixo risco. Em contrapartida, no grupo IP, a média da classificação de risco atribuída às mulheres passou de baixo para alto.

Pode-se inferir que os modelos de intervenção realizados nos grupos II e IG, foram semelhantes na redução do IC-1, mesmo apresentando propostas de intervenção diferentes. Ambas apresentaram redução nos valores iniciais, enquanto que o modelo de intervenção padrão não demonstrou redução favorável. 
Tabela 1. Tabela de caracterização da amostra

\begin{tabular}{lcccc}
\hline \multicolumn{1}{c}{ Variável } & $\begin{array}{c}\text { IP } \\
(\mathbf{n}=\mathbf{1 6})\end{array}$ & $\begin{array}{c}\text { IG } \\
(\mathbf{n}=\mathbf{2 0})\end{array}$ & $\begin{array}{c}\text { II } \\
(\mathbf{n = 2 3})\end{array}$ & $* \boldsymbol{p}$ \\
\hline Peso (kg) & $97,05 \pm 14,96$ & $91,04 \pm 12,22$ & $91,60 \pm 13,55$ & $>0,05$ \\
IMC $\left(\mathrm{kg} / \mathrm{m}^{2}\right)$ & $34,10 \pm 4,32$ & $33,89 \pm 3,48$ & $32,31 \pm 2,94$ & $>0,05$ \\
CA (cm) & $113,41 \pm 9,62$ & $110,04 \pm 10,27$ & $110,65 \pm 6,89$ & $>0,05$ \\
Glicemia (mg/dL) & $121,56 \pm 45,01$ & $107,90 \pm 42,56$ & $122,50 \pm 44,96$ & $>0,05$ \\
PAS (mmHg) & $130,75 \pm 10,73$ & $127,90 \pm 12,77$ & $129,50 \pm 12,61$ & $>0,05$ \\
PAD (mmHg) & $86,38 \pm 9,33$ & $85,50 \pm 9,10$ & $85,00 \pm 9,88$ & $>0,05$ \\
Colesterol total (mg/dL) & $197,94 \pm 44,17$ & $220,00 \pm 66,10$ & $219,00 \pm 50,09$ & $>0,05$ \\
Triglicerídeos (mg/dL) & $171,06 \pm 84,28$ & $220,75 \pm 111,04$ & $288,00 \pm 89,06$ & $>0,05$ \\
HDL-c (mg/dL) & $48,13 \pm 11,52$ & $49,45 \pm 11,32$ & $34,50 \pm 14,61$ & $>0,05$ \\
LDL-c (mg/dL) & $115,60 \pm 47,42$ & $126,40 \pm 55,75$ & $126,90 \pm 50,46$ & $>0,05$ \\
\hline
\end{tabular}

IP = Intervenção Padrão; IG = Intervenção em Grupo; II = Intervenção Individual; IMC: Índice de Massa Corporal; CA: Circunferência Abdominal; PAS: Pressão Arterial Sistólica; PAD: Pressão Arterial Diastólica; HDL: Colesterol HDL; LDL: Colesterol LDL. *Análises estatísticas realizadas para verificar homogeneidade da amostra.

Tabela 2. Tabela efeito das diferentes intervenções para a modificação do estilo de vida sobre o IC-1

\begin{tabular}{lcccc}
\hline Grupo & $\begin{array}{c}\text { Antes da } \\
\text { intervenção }\end{array}$ & $\begin{array}{c}\text { Após a } \\
\text { intervenção }\end{array}$ & Variação absoluta & Variação percentual (\%) \\
\hline IP $(\mathbf{n}=\mathbf{1 6})$ & $4,23 \pm 0,95$ & $4,84 \pm 0,94$ & 0,61 & 14,00 \\
IG $(\mathbf{n}=\mathbf{2 0})$ & $4,58 \pm 1,46$ & $4,35 \pm 1,33$ & $-0,23^{*}$ & $-5,02$ \\
II $(\mathbf{n}=\mathbf{2 3})$ & $5,37 \pm 1,70$ & $4,68 \pm 1,16$ & $-0,70^{*}$ & $-12,84$ \\
\hline
\end{tabular}

Os dados representam a média e desvio padrão da média, a variação absoluta e percentual. IP = intervenção padrão; IG $=$ intervenção em grupo; II = intervenção individual. * = Diferença significativa quando comparado ao momento pré-intervenção em cada grupo. Teste de análise de variância e Post-Hoc teste de Tukey HSD.

Tabela 3. Tabela efeito das diferentes propostas de intervenções para a modificação do estilo de vida sobre a classificação do IC-1

\begin{tabular}{cccccccc}
\hline \multirow{2}{*}{ Gênero } & & \multicolumn{2}{c}{ IP $(\mathbf{n}=\mathbf{1 6})$} & & IG $(\mathbf{n}=\mathbf{2 0})$ & II $(\mathbf{n}=\mathbf{2 3})$ & \\
\cline { 2 - 8 } & & IC-1 & Classificação & IC-1 & Classificação & IC-1 & Classificação \\
\hline \multirow{2}{*}{ Masculino } & Pré & $4,60 \pm 0,80$ & Baixo risco DCV & $5,30 \pm 1,63$ & Alto risco DCV & $6,73 \pm 1,02$ & Alto risco DCV \\
& Pós & $4,88 \pm 0,85$ & Baixo risco DCV & $4,76 \pm 1,58$ & Baixo risco DCV* & $5,47 \pm 0,70$ & Alto risco DCV \\
\hline \multirow{2}{*}{ Feminino } & Pré & $3,61 \pm 0,89$ & Baixo risco DCV & $3,98 \pm 1,03$ & Baixo risco DCV & $4,65 \pm 1,54$ & Alto risco DCV \\
& Pós & $4,76 \pm 1,16$ & Alto risco DCV* & $4,01 \pm 1,04$ & Baixo risco DCV & $4,25 \pm 1,14$ & Baixo risco DCV*
\end{tabular}

Os valores representam a média e o desvio padrão da média do IC-1 avaliados nos momentos pré e pós-intervenções. A classificação de risco foi determinada a partir dos pontos de corte estabelecidos em função do sexo. IP = Intervenção Padrão; IG = Intervenção em Grupo; II = Intervenção Individual. DCV: Doença Cardiovascular. *Variação em relação à classificação de risco do IC-1. 


\section{DISCUSSÃO}

Neste estudo, as intervenções em grupo e individual mostraram efeito na redução do IC-1 em indivíduos com SM. Assim, o impacto positivo reforça a importância da modificação do estilo de vida na prevenção, controle e tratamento dos fatores de risco cardiometabólicos, conforme também relatos na literatura ${ }^{22-26}$.

O IC-1 é descrito como uma ferramenta útil para avaliar risco de desenvolver doenças cardiovasculares ${ }^{11}$,

${ }^{12}$ na prática clínica, porém poucos estudos descrevem o comportamento do IC-1 na SM, bem como o efeito de programas de intervenção sobre esse índice.

Estudo realizado por Castelli ${ }^{12}$ verificou que $30 \%$ dos pacientes que necessitaram de intervenção cirúrgica após eventos coronarianos, apresentavam valores IC-1 acima da média recomendada. Castelli propôs ainda, que se a relação colesterol total/HDL-c estiver acima de $4 \mathrm{mg} /$ $\mathrm{dL}$, deve-se inicialmente avaliar os valores absolutos de triglicerídeos, pois esse interfere na quantidade total de colesterol plasmático. Nesse estudo, observamos que a maioria dos indivíduos no início da intervenção apresentava uma classificação de alto RCV segundo o IC-1, e ao serem submetidos à intervenção multidisciplinar e incentivados a modificar seus hábitos de vida, melhoraram os parâmetros que compõe este índice, já que os indivíduos apresentaram uma mudança de $-5,02 \%$ e $-12,84 \%$ nos grupos IG e II, respectivamente.

Assim, estes achados demonstram que uma alimentação equilibrada e a adesão a uma vida fisicamente ativa são metas fundamentais para indivíduos com SM e constituem dois eixos fundamentais no processo de modificação do estilo de vida para a melhora dos fatores de risco modificáveis para DCV ${ }^{22,26}$. Estudos têm comprovado que o exercício físico possui um importante papel na manutenção da estrutura e função vascular ${ }^{8}$, e a inadequação alimentar já se mostrou impactar de forma significativamente negativa sobre mortalidade cardiovascular ${ }^{8,24,27}$.

A interação entre os diferentes tipos de lipídios séricos e sua relação com eventos cardiovasculares permanecem sendo um desafio para a área da saúde. $\mathrm{O}$ aumento da relação colesterol total/HDL-c, está relacionado ao maior RCV, pois frequentemente está associado à hipertrigliceridemia, hipertensão arterial, intolerância à glicose e resistência à insulina ${ }^{8,10,14,28,29}$, todos estes componentes importantes no desenvolvimento da SM e com alta prevalência nos indivíduos estudados. Além disso, o aumento do colesterol total sérico é considerado um fator independente para o desenvolvimento de doenças ateroscleróticas e coronarianas, principalmente por sua relação com a inflamação e a instabilidade da placa de ateroma $^{8,30}$. Assim, a redução do nível de colesterol total se apresenta como uma importante meta terapêutica. No início da intervenção, os indivíduos dos três grupos apresentam valores de colesterol totais séricos limítrofes ou acima do recomendado ${ }^{8}$ e uma vez que, os homens do grupo IG e as mulheres do grupo II obtiveram mudanças significativas nos valores dos componentes que compõe o IC-1 e consequentemente modificaram sua classificação de risco. A partir dos resultados encontrados podemos identificar que mesmo intervenções multidisciplinares de curta duração (03 meses) são capazes de produzir mudanças positivas.

Rankin et al. ${ }^{26}$ obtiveram resultados semelhantes ao do presente estudo, em que uma intervenção para modificação do estilo de vida com duração de 30 dias foi capaz de reduzir significativamente os índices de massa corporal, PAS, PAD, colesterol total, triglicerídeos e glicemia de jejum, reafirmando que mudanças significativas nos fatores de risco de DCV podem ser alcançadas com intervenções de curto prazo.

Corroborando os dados encontrados, Santos et al. ${ }^{31}$ propuseram um trabalho que tinha por objetivo avaliar a eficácia de programas de conscientização sobre o controle de fatores de risco para DCV. Dentre as variáveis estudadas os autores verificaram que o IC-1 reduziu de forma mais intensa no grupo que participou de consultas de conscientização quando comparados aos demais voluntários alocados no grupo que recebeu apenas orientações padrão, o que novamente reforça a ideia de que abordagens multidisciplinares impactam de forma positiva e significativa no processo de mudança para promoção da saúde em indivíduos em potencial risco para desenvolvimento de DCV.

\section{CONCLUSÃO}

O presente estudo demonstrou que intervenções multidisciplinares para modificação do estilo de vida são capazes de produzir efeito benéfico na redução do IC-1, reduzindo os fatores de risco para DCV passíveis de modificação em indivíduos com SM. Logo, a adoção de hábitos de vida considerados saudáveis, como prática regular de 
exercício físico e alimentação balanceada, deve ser incentivada nessa população a fim de evitar possíveis desfechos cardiovasculares.

\section{REFERÊNCIAS}

1. Brandão AP, Brandão AA, Nogueira AR, Suplicy $H$, Guimarães JI, Oliveira JE et al. Sociedade Brasileira de Cardiologia. I Diretriz brasileira de diagnóstico e tratamento da síndrome metabólica. Arq Bras Cardiol. 2005; 84(supl1): 1-28.

2. Leite PB, Dâmaso AR, Poli VS, Sanches RB, Silva SGA, Fidalgo JPN et al. Long-term interdisciplinary therapy decreases symptoms of binge eating disorder and prevalence of metabolic syndrome in adults with obesity. Nutr. res. 2017 Apr;40:57-64.

3. Mottillo S, Filion KB, Genest J, Joseph L, Pilote $\mathrm{L}$, Poirier $\mathrm{P}$ et al. The metabolic syndrome and cardiovascular risk: a systematic review and metaanalysis. J Am Coll Cardiol. 2010 Sep;56(14):1113-32.

4. Bortoletto MSS, Souza RKT, Cabrera MAS, González AD. (2014). Síndrome metabólica em estudos com adultos brasileiros: uma revisão sistemática. Rev Saúde Pub PR. 2014;15(4): 86-98

5. Malta DC, Andrade SC, Claro RM, Bernal RTI, Monteiro CA. Evolução anual da prevalência de excesso de peso e obesidade em adultos nas capitais dos 26 estados brasileiros e no Distrito Federal entre 2006 e 2012. Rev Bras Epidemiol. 2014;17 (Suppl 1):267-76.

6. Baez-Duarte BG, Zamora-Gínez I, González-Duarte R, Torres-Rasgado E, Ruiz-Vivanco G, Pérez-Fuentes R et al. Triglyceride/high-density lipoprotein cholesterol (TG/HDL-C) index as a reference criterion of risk for metabolic syndrome (MetS) and low insulin sensitivity in apparently healthy. Gac Med Mex. 2017 Mar -Apr;153(2):152-58.

7. Saboya PP, Bodanese LC, Zimmermann PR, Gustavo AS, Macagnan FE, Feoli AP et al. Intervenção de Estilo de Vida na Síndrome Metabólica e seu Impacto na
Qualidade de Vida: Um Estudo Controlado Randomizado. Arq Bras Cardiol. 2016 Sep;108(1):60-9.

8. Faludi AA, Izar MCO, Saraiva JFK, Chacra APM, Bianco HT, Neto AA et al. Atualização da Diretriz Brasileira de Dislipidemias e Prevenção da Aterosclerose. Arq Bras Cardiol. 2017 Aug;109(2Supl.1):1-76

9. Murguía-Romero M, Jiménez-Flores JR, Sigrist-Flores SC, Espinoza-Camacho MA, Jiménez-Morales M, Piña E et al. Plasma triglyceride/HDL-cholesterol ratio, insulin resistance, and cardiometabolic risk in young adults.J Lipid Res. 2013 Oct;54(10):2795-9.

10. Mozaffarian D, Benjamin EJ, Go AS, Arnett DK, Blaha MJ, Cushman $M$ et al. Executive summary: Heart disease and stroke statistics-2016 update: A Report from the American Heart Association. Circulation. 2016. 133(4):447-454.

11. Castelli WP. Lipids, risk factors and ischaemic heart disease. Atherosclerosis. 1996;124:S1-S9.

12. Castelli WP, Garrison RJ, Wilson PW, Abbott RD, Kalousdian S, Kannel WB. Incidence of coronary heart disease and lipoprotein cholesterol levels. The Framingham Study. JAMA. 1986;256: 2835-8.

13. Program CEN. Expert Panel on Detection, Evaluation, and Treatment of High Blood Cholesterol in Adults (Adult Treatment Panel III): Third Report of the National Cholesterol Education Program (NCEP) Expert Panel on Detection.Evaluation, and Treatment of High Blood Cholesterol in Adults (Adult Treatment Panel III) final report. Circulation. 2002;106(25):3143-421

14. Organization $W_{H}$. Diet, nutrition and the prevention of chronic diseases. WHO: 2002; 916.

15. Velasquez MM, DiClemente CC, Crouch C, Stephens NS. Group treatment for substance abuse: A stages-of-change therapy manual. Guilford Publications. 2015.

16. Witschi J, Porter D, Vogel S, Buxbaum R, Stare FJ, Slack W. A computer-based dietary counseling system. J Am Diet Assoc. 1976;69:385-90. 
17. Willett WC, Sampson L, Stampfer MJ, Rosner B, Bain $\mathrm{C}$, Witschi J et al. Reproduciblity and validity of a semiquantitative food frequency questionnaire. Am J Epidemiol. 2017;185(11): 1109-23.

18. Lohman TG, Roche AF, Martorell R. Anthropometric standardization reference manual. Champaign (IL): Human Kinetics Books; 1988.

19. World Health Organization. Obesity: preventing and managing the global epidemic. WHO: 2000. p. 256, n. 284.

20. Malachias MVB, Souza WKSB, Plavnik FL, Rodrigues CIS, Brandão AA, Neves MFT, et al. $7^{\mathrm{a}}$ Diretriz Brasileira de Hipertensão Arterial. Arq Bras Cardiol. 2016; 107

21. Friedewald WT, Levy RI, Fredrickson DS. Estimation of the Concentration of Low-Density Lipoprotein Cholesterol in Plasma, without Use of the Preparative Ultracentrifuge. ClinChem. 1972; 18:6.

22. Crovetto M, Valladares M, Espinoza V, Mena F, Oñate G, Fernandez M. Effect of healthy and unhealthy habits on obesity: a multicentric study. Nutrition. 2018; 54:7-11.

23. Li XT, Liao W, Yu HJ, Liu MW, Yuan S, Tang BW. Combined effects of fruit and vegetables intake and physical activity on the risk of metabolic syndrome among Chinese adults. PLoS One. 2017;12(11): e0188533.

24. Farhangi MA, Jahangiry L. Dietary diversity score is associated with cardiovascular risk factors and serum adiponectin concentrations in patients with metabolic syndrome. BMC Cardiovasc Disord. 2018; 18(1): 68.

25. Phing CH, Saad HA, Nisak M.B, Nasir MM. Effectiveness of physical activity intervention among government employees with metabolic syndrome. J Exerc Sci Fit. 2017; 15(2):55-62.

26. Rankin P, Morton DP, Diehl H, Gobble J, Morey P, Chang E. Effectiveness of a volunteer-delivered lifestyle modification program for reducing cardiovascular disease risk factors. Am J Cardiol. 2012;109 (1): 82-6.

27. Suliga E, Kozieł D, Cieśla E, Rębak D, Głuszek S. Dietary Patterns in Relation to Metabolic Syndrome among Adults in Poland: A Cross-Sectional Study. Nutrients. 2017; 9(12):1366.

28. Furukawa S, Fujita T, Shimabukuro M, Iwaki M, Yamada $Y$, Nakajima $Y$ et al. Increased oxidative stress in obesity and its impact on metabolic syndrome. J Clin Invest. 2017;114(12):1752-61.

29. Yubero-Serrano EM, Delgado-Lista J, Tierney AC, Perez-Martinez P, Garcia-Rios A, Alcala-Diaz JFet al. Insulin resistance determines a differential response to changes in dietary fat modification on metabolic syndrome risk factors: the LIPGENE study. Am J Clin Nutr. 2015 Dec;102(6):1509-17.

30. Peters SA, Singhateh Y, Mackay D, Huxley RR, Woodward M. Total cholesterol as a risk factor for coronary heart disease and stroke in women compared with men: A systematic review and meta-analysis. Atherosclerosis. 2016;248:123-31.

31. Santos RD, Nascimento LO, Maranhão R. Avaliação dos efeitos a curto prazo de programas de conscientização e de terapia com pravastatina em indivíduos de alto risco para doença cardiovascular provenientes de consultórios particulares. Arq Bras Cardiol. 1997; 69(4):225-30.

Recebido em: 10/07/2018

Aceito em: 20/09/2018 